\title{
Association of aspartate and alanine transaminases with dyslipidemia in newly diagnosed and long duration of type 2 diabetes mellitus subjects.
}

1. M.Phil, Ph.D Scholar Clinical Biochemistry and

Psychopharmacology Research Unit Department of Biochemistry University of Karachi-Pakistan

2. Ph.D

Proefssor \& Head of Biochemistry Clinical Biochemistry and Psychopharmacology Research Unit, Department of Biochemistry University of Karachi-Pakistan.

3. Ph.D

Professor \& Head of Biochemistry Baqai Medical University Karachi. Research Director (Honorary) Baqai Institute of Diabetology and Endocrionology

Baqai Medical University, Karachi.

4. FRCP

Professor Medicine

Baqai Institute of Diabetology and Endocrinology,

Baqai Medical University, KarachiPakistan.

\section{Correspondence Address:}

Dr. Nazish Waris

Clinical Biochemistry and

Psychopharmacology Research Unit

Department of Biochemistry, University of Karachi-Pakistan.

nwaris@bide.edu.pk.

Article received on:

$12 / 02 / 2021$

Accepted for publication:

23/05/2021

Nazish Waris 1 , Samina Bano ${ }^{2}$, Asher Fawwad ${ }^{3}$, Abdul Basit ${ }^{4}$

ABSTRACT... Objective: Association between alanine transaminase (ALT) and aspartate transaminase (AST) with newly diagnosed and known type 2 diabetes mellitus (DM) and to estimate association of liver enzymes with lipid profile in type 2 DM subjects. Study Design: Prospective Clinical study. Setting: Karachi University with Collaboration of Baqai Institute of Diabetology and Endocrinology. Period: November 2018 to May 2019. Material \& Methods: Total 100 people were divided into four groups; Group I: 25 healthy controls with normal glucose tolerance, Group II: 25 newly diagnosed DM, Group III: 25 known DM type 2 with $<5 y e a r s$ duration and Group IV: 25 known DM type 2 between 5-10 years duration. Baseline data was collected on predesigned questionnaire. Blood samples for biochemical parameters were analyzed using standardized laboratory techniques. Results: Group I mean age (years)

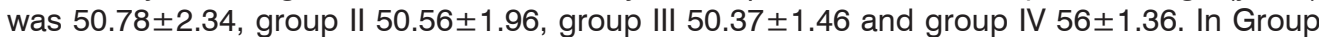
I, ALT and AST were significantly correlated to each other's. In group II, ALT was significantly correlated with AST, triglycerides and HDL-C, while AST correlated with ALT and HDL-C. In group III, ALT was significantly correlated with AST, while AST correlated to ALT, triglycerides and $\mathrm{HbA1c}$. However, in group IV, ALT was significantly correlated with AST, LDL-C and HDL-C, and, AST with ALT, total cholesterol, LDL-C and HDL-C. Conclusion: Elevated ALT and AST- the salient markers for disease of non-alcoholic fatty liver with deranged dyslipidemia were found in known type 2 DM as well as in newly diagnosed type 2 DM subjects.

\section{Key words: $\quad$ ALT, AST, Dyslipidemia, Duration, Type 2 DM.}

Article Citation: Waris N, Bano S, Fawwad A, Basit A. Association of aspartate and alanine transaminases with dyslipidemia in newly diagnosed and long duration of type 2 diabetes mellitus subjects. Professional Med J 2021; 28(12):17111717. https://doi.org/10.29309/TPMJ/2021.28.12.6421

\section{INTRODUCTION}

The International Diabetes Federation has estimated the increasing prevalence of type 2 diabetes mellitus (DM) to be 463 million in 2019 and projected to reach 578 million by 2030 , and 700 million by 2045 , worldwide. ${ }^{1}$ The recent second National Diabetes Survey of Pakistan (2016-2017) has estimated the current prevalence of type 2 DM as high as $26.3 \%$ including newly diagnosed type 2 DM $7.1 \%{ }^{2}$

Many studies have been reported that type 2 DM is associated with a number of liver abnormalities, such as abnormal glycogen deposition, nonalcoholic fatty liver disease (NAFLD), fibrosis, cirrhosis, hepatocellular carcinomas, abnormal elevated hepatic enzymes, acute liver disease, viral hepatitis and related metabolic syndrome. ${ }^{3,4}$
Abnormal levels of liver enzymes, particularly aminotransferases are commonly used in clinical setting to diagnose liver dysfunctions. Elevated alanine trans aminase (ALT) to aspartate trans aminase (AST) ratio are two important prognostic features of NAFLD and metabolic disorders such as insulin resistance in type 2 DM subjects. ${ }^{5,6}$ Although, the pathogenesis is unclear, hyperinsulinemia as a result of insulin resistance is thought to play an important role in pancreatic enzyme dysfunction in type $2 \mathrm{DM}$ along with hepatic triglyceride accumulation and defective lipid metabolism. ${ }^{7}$ Altered lipoprotein metabolism and derangement liver enzymes may be predisposing and have been identified as an independent risk factors for progression of cardiovascular disease and kidney disease in type 2 DM..$^{8,9}$ Moreover, a study in a white and African- 
American population found that $\mathrm{ALT} \geq 26 \mathrm{IU} / \mathrm{L}$ increases the diabetes prediction substantially. ${ }^{10}$

To our knowledge, limited studies have demonstrated the relationship of ALT and AST with diabetes in Pakistan. Thus, this study is designed to examine the association between ALT and AST with newly diagnosed diabetes (NDD) and known type 2 DM and to estimate association of liver enzymes with lipid profile in type 2 DM subjects.

\section{MATERIAL \& METHODS}

This prospective clinical study was planned at Department of Biochemistry, University of KarachiPakistan with collaboration of Baqai Institute of Diabetology and Endocrinology (BIDE), Baqai Medical University, Karachi-Pakistan. Subjects visiting the outpatient department of BIDE were recruited between November 2018 to May 2019. Total 100 subjects were included, categorized into four groups; Group I: includes 25 control subjects with normal glucose tolerance, Group II: 25 subjects with NDD, Group III: 25 subjects with $<5$ years duration of known type $2 \mathrm{DM}$ and Group IV: 25 subjects between 5-10 years duration of type 2 DM. Ethical approval was obtained from Institutional Review Board of BIDE (IRB no.: BIDE/ IRB/NWARIS/10/26/18/0206).

Subjects with NDD (fasting plasma glucose (FPG) level $\geq 126 \mathrm{mg} / \mathrm{dL}$ or 2-hour post glucose level $\geq 200 \mathrm{mg} / \mathrm{dL}$ or both.) and controls with normal glucose tolerance (FPG levels below $100 \mathrm{mg} / \mathrm{dL}$ and post glucose levels below $140 \mathrm{mg} / \mathrm{dL}$ ) without any anti diabetic medications were diagnosed according to world health organization criteria. Subjects taking anti-diabetic medications with or without using statins were enrolled as known diabetes. ${ }^{11}$ Exclusion criteria involved individuals who did not agree to participate, severe renal or hepatic dysfunction, excessive alcohol intake, dehydration, diarrhea or vomiting, gestational diabetes, type $1 \mathrm{DM}$ and impaired type $2 \mathrm{DM}$. Subjects with NDD and healthy controls using lipid lowering agents such as statins were also excluded.

Following inclusion criteria, baseline demographic and anthropometric data was collected from each subject on predesigned questionnaire after obtaining informed consent. To confirm NDD and controls with normal glucose tolerance, blood samples were drawn before and after post glucose level at 120 minutes at specified tubes. For subjects with known diabetes, fasting and random blood samples were drawn for biochemical parameters. Blood samples for $\mathrm{HbA} 1 \mathrm{c}$, lipid profile, liver enzymes as ALT and AST were also obtained. Standardized techniques were used for biochemical analysis and to measure height, body weight and blood pressure.

\section{Statistical Analysis}

Data was presented as mean \pm SEM (standard error of the mean) or $n$ (\%). Statistical analysis was performed using one-way ANOVA and followed by post hoc analysis (Least Significant Difference (LSD) test) to establish which means differed significantly. Z-test for two population proportion tests was used to check whether two groups differ significantly on some single categorical characteristic. All values are presented as mean \pm SEM or $n(\%)$ for each group. For significance of difference, group II compared by group I is indicated by "a"; group III compared by group I and II is indicated by "a" and "b", respectively; group IV compared by group I, II and III is indicated by "a", "b" and "c", respectively. Pearson's correlation was performed to see the relationship of AST and ALT with various parameters. Significance of difference is considered by $p<0.05$. All statistical analyses were done using statistical package of social sciences version 20 .

\section{RESULTS}

Mean age of group I was $50.78 \pm 2.34$ years, group II was $50.56 \pm 1.96$ years, group III was $50.37 \pm 1.46$ years and group IV was $56 \pm 1.36$ years. Details for anthropometric and various biochemical parameters are shown in Table-l. AST and ALT levels were significantly higher in known type 2 DM as well as NDD subjects compared to controls. Most of the subjects with known diabetes in group III 19(76\%) and group IV 25(100\%) were using statins.

Table-Il categorize the dyslipidemic parameters 
using their standard cutoff values in all groups. Hypercholesterolemia $\geq 200$ was found $11(44 \%)$ in healthy control, $10(40 \%)$ in NDD, 5(20\%) in $<5$ years duration of diabetes and $8(32 \%)$ in 5-10 years duration of diabetes similarly, hypertriglyceridemia $\geq 150$ was observed in 9(36\%), 14(56\%), 18(72\%) and 12(48\%) subjects, respectively. LDL-C $\geq 130$ was found in $7(28 \%)$, 10(40\%), 3(12\%) and 8(32\%) and HDL-C <50/40 was found in 23(92\%), 19(76\%), 20(20\%) and $19(76 \%)$ subjects in group I, II, III and IV, respectively. AST $\geq 35$ was significantly found higher in group IV, group III and II, respectively, compared to group I. Meanwhile, ALT was also to group II and group I, respectively.

Table-III reveals the correlation between ALT and AST and lipid profile in group I, II, III and IV, respectively. In Group I, ALT and AST were significantly correlated to each other's. In group II, ALT was significantly correlated with AST, triglycerides and HDL-C, while AST significantly correlated with ALT and HDL-C. In group III, ALT significantly higher in group IV and III, compared

was significantly correlated with AST, while AST significantly correlated to ALT, triglycerides and HbA1c. However, in group IV, ALT was significantly correlated with AST LDL-C and HDL-C, while, AST significantly correlated with ALT, total cholesterol, LDL-C and HDL-C.

All values are presented as mean \pm SEM for each group. For significance of difference group II compared by group I is indicated by "a"; group III compared by group I and II is indicated by "a" and "b", respectively; group IV compared by group I, II and III is indicated by "a", "b" and "c", respectively. Significance of difference is considered by $\mathrm{p}<0.05$.

All values are presented as $n(\%)$ for each group. For significance of difference group II compared by group I is indicated by "a"; group III compared by group I and II is indicated by "a" and " $b$ ", respectively; group IV compared by group I, II and III is indicated by "a", "b" and "c", respectively. Significance of difference is considered by $p<0.05$.

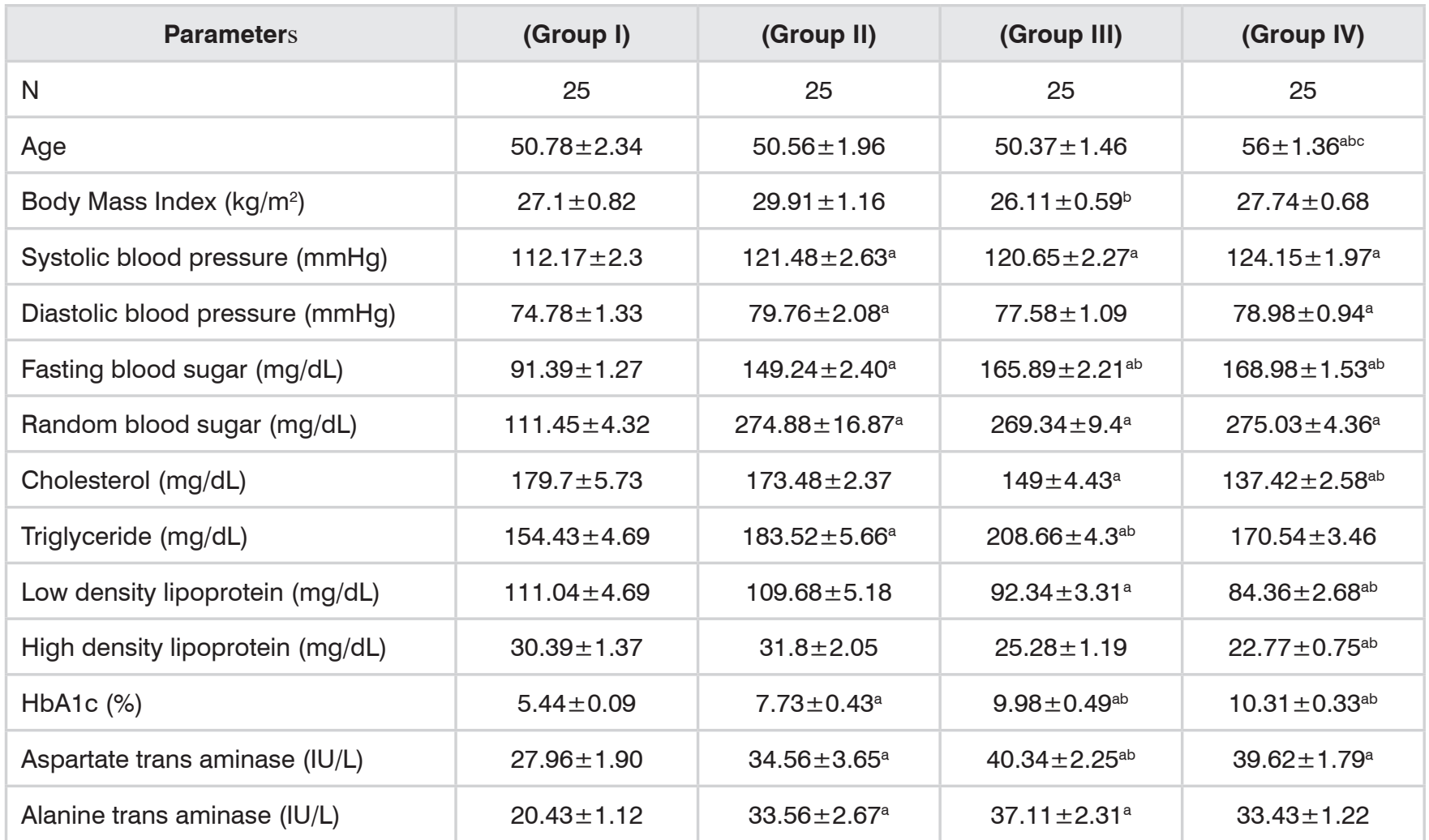

Table-I. Various biochemical parameters of healthy control and diabetes groups. 


\begin{tabular}{|c|c|c|c|c|}
\hline Parameters & (Group I) & (Group II) & (Group III) & (Group IV) \\
\hline \multicolumn{5}{|c|}{ Cholesterol (mg/dL) } \\
\hline$<200$ & $14(56 \%)$ & $15(60 \%)$ & $20(80 \%)$ & $17(68 \%)$ \\
\hline \multicolumn{5}{|c|}{ Triglyceride (mg/dL) } \\
\hline$<150$ & $16(64 \%)$ & $11(44 \%)$ & $7(28 \%)$ & $13(52 \%)$ \\
\hline$\geq 150$ & $9(36 \%)$ & $14(56 \%)^{\mathrm{a}}$ & $18(72 \%)^{a b}$ & $12(48 \%)$ \\
\hline$<130$ & $18(72 \%)$ & $15(60 \%)$ & $22(88 \%)$ & $17(68 \%)$ \\
\hline$\geq 130$ & $7(28 \%)$ & $10(40 \%)$ & $3(12 \%)^{\mathrm{ab}}$ & $8(32 \%)^{\mathrm{ab}}$ \\
\hline \multicolumn{5}{|c|}{ High density lipoprotein (mg/dL) } \\
\hline$<50 / 40$ & $2(8 \%)$ & $6(24 \%)$ & $5(80 \%)$ & $6(24 \%)$ \\
\hline$\geq 50 / 40$ & $23(92 \%)$ & $19(76 \%)$ & $20(20 \%)$ & $19(76 \%)$ \\
\hline \multicolumn{5}{|c|}{ Alanine trans aminase (IU/L) } \\
\hline$<35$ & $21(84 \%)$ & $20(80 \%)$ & $15(60 \%)$ & $14(56 \%)$ \\
\hline$\geq 35$ & $4(16 \%)$ & $5(20 \%)$ & $10(40 \%)^{a b}$ & $11(44 \%)^{a}$ \\
\hline
\end{tabular}

Table-II. Anthropometric and biochemical parameters cutoffs of healthy control and diabetes groups.

\begin{tabular}{|c|c|c|c|c|c|}
\hline Groups & Parameters & ALT & P-Value & AST & P-Value \\
\hline \multirow{7}{*}{ Group 1} & Alanine trans aminase (IU/L) & 1 & - & 0.49 & 0.018 \\
\hline & Aspartate trans aminase (IU/L) & 0.49 & 0.018 & 1 & - \\
\hline & Cholesterol (mg/dL) & 0.092 & 0.676 & 0.14 & 0.525 \\
\hline & Triglyceride (mg/dL) & 0.144 & 0.513 & 0.041 & 0.852 \\
\hline & Low density lipoprotein (mg/dL) & -0.018 & 0.935 & -0.011 & 0.959 \\
\hline & High density lipoprotein (mg/dL) & 0.229 & 0.293 & 0.228 & 0.294 \\
\hline & $\mathrm{HbA} 1 \mathrm{c}(\%)$ & 0.073 & 0.74 & 0.091 & 0.679 \\
\hline \multirow{7}{*}{ Group 2} & Alanine trans aminase (IU/L) & 1 & - & 0.849 & $<0.0001$ \\
\hline & Aspartate trans aminase (IU/L) & 0.849 & $<0.0001$ & 1 & - \\
\hline & Cholesterol (mg/dL) & -0.149 & 0.476 & -0.179 & 0.393 \\
\hline & Triglyceride (mg/dL) & 0.418 & 0.037 & 0.353 & 0.083 \\
\hline & Low density lipoprotein (mg/dL) & -0.047 & 0.822 & -0.209 & 0.317 \\
\hline & High density lipoprotein (mg/dL) & -0.58 & 0.002 & -0.484 & 0.014 \\
\hline & $\mathrm{HbA} 1 \mathrm{c}(\%)$ & -0.214 & 0.315 & -0.133 & 0.534 \\
\hline \multirow{7}{*}{ Group 3} & Alanine trans aminase (IU/L) & 1 & - & 0.755 & $<0.0001$ \\
\hline & Aspartate trans aminase (IU/L) & 0.755 & $<0.0001$ & 1 & - \\
\hline & Cholesterol $(\mathrm{mg} / \mathrm{dL})$ & 0.071 & 0.714 & 0.073 & 0.707 \\
\hline & Triglyceride (mg/dL) & 0.258 & 0.177 & 0.242 & 0.020 \\
\hline & Low density lipoprotein (mg/dL) & 0.024 & 0.904 & -0.007 & 0.973 \\
\hline & High density lipoprotein (mg/dL) & 0.042 & 0.827 & 0.069 & 0.722 \\
\hline & $\mathrm{HbA} 1 \mathrm{c}(\%)$ & -0.294 & 0.115 & -0.348 & 0.059 \\
\hline \multirow{7}{*}{ Group 4} & Alanine trans aminase (IU/L) & 1 & - & 0.732 & $<0.0001$ \\
\hline & Aspartate trans aminase (IU/L) & 0.732 & $<0.0001$ & 1 & - \\
\hline & Cholesterol (mg/dL) & -0.245 & 0.066 & -0.323 & 0.014 \\
\hline & Triglyceride (mg/dL) & -0.083 & 0.538 & -0.149 & 0.269 \\
\hline & Low density lipoprotein (mg/dL) & -0.292 & 0.025 & -0.453 & $<0.0001$ \\
\hline & High density lipoprotein (mg/dL) & -0.291 & 0.028 & -0.318 & 0.016 \\
\hline & $\mathrm{HbA} 1 \mathrm{c}(\%)$ & -0.128 & 0.355 & -0.204 & 0.138 \\
\hline
\end{tabular}

Table-III. Correlation coefficient between liver enzymes and other studied parameters of Group I, Group II, Group III and Group IV. 
Values are presented in correlation coefficient (r) to see the relationship of AST and ALT with studied parameters. Significance of difference is considered by $\mathrm{p}<0.05$.

\section{DISCUSSION}

In this study, significantly increased AST and ALT levels with deranged dyslipidemia were observed with long duration of type 2 DM subjects. Similarly, ALT and AST levels were also significantly higher in NDD subjects with hypertriglyceridemia compared to controls with normal glucose tolerance.

Significant correlation of elevated liver enzymes with deranged dyslipidemia in type 2 DM were similar to recent study. ${ }^{12}$ Elevated ALT and AST as a significant predictor of diabetes are to some extent in agreement with earlier study. ${ }^{13}$ Cho et al., observed increased activity of liver enzymes, markedly increase ALT was associated with twofold increase in type 2 DM risk independently of conventional risk factors and serve as a useful marker to identify individuals at high risk of type 2 DM in Asian populations. ${ }^{14}$ In this study, AST upper limit $>35$ was found higher compared to ALT opposed to recent study that showed low AST levels compared to ALT levels in type 2 DM. ${ }^{15}$ Meanwhile, elevated ALT and AST in NDD subjects as compared to healthy subjects were also observed showing that in asymptomatic individuals with mild elevations of ALT and AST, $98 \%$ have probable risk of liver disease commonly fatty liver disease consistent with previous study. 8,16

In our study, deranged liver enzymes were investigated with dyslipidemia in type $2 \mathrm{DM}$ subjects similar to study from Nigeria. ${ }^{17}$ In previous study, insulin resistance, hypertriglyceridemia, and hypercholesterolemia were described as a cause of NAFLD and were reported in around $40-70 \%$ diabetic subjects. ${ }^{8}$ Hyperlipidemia in terms of hypercholesterolemia and elevated LDL-C were significantly found lower in known type 2 DM subjects compared to NDD and healthy individuals in this study. However, hypertriglyceridemia and low HDL-C were nonsignificantly higher in <5years duration of known DM subjects. Our results show that either known
DM subjects were using lipid controlling drugs (statins), but results for hypertriglyceridemia and low HDL-C levels are consistent with the study of Han Ni et al., who reported elevated determinants of liver function tests with hyperlipidemia in type 2 DM. ${ }^{18}$ Our results are also consistent with Saligram et al., study, who discussed the association of increased ALT levels with elevated triglycerides and low HDL-C. ${ }^{19}$ Moreover, oral antidiabetic agents, poor glycemic control and statins are also considered as a cause of deranged liver enzymes with histological changes in liver. Deranged liver enzymes were also observed with poor glycemic status in type 2 DM subjects in this study likely to Bora et al., study. ${ }^{20}$ Asians at lower body mass index develop type 2 DM compared to western populations. It was also suggested that liver may play a vital role in development of type 2 DM in relatively lean Asian populations. ${ }^{10} \mathrm{We}$ also observed non-significantly high body mass index in all groups that was comparable to study conducted in India. ${ }^{21}$

Liver imaging and histopathology of liver biopsy were not done are the limitations of this study. But, comparing ALT and AST with dyslipidemia in NDD and long duration of type $2 \mathrm{DM}$ has unmasked some important and relevant information about the impact of DM on the liver may increase worth to literature. Routine screening of ALT and AST with dyslipidemic profile in type 2 DM subjects may assist early detection of liver abnormalities and arrest the progress of disease to chronic conditions. Further studies need to be done along with the assessment of blood coagulation and histopathology of liver biopsy.

\section{CONCLUSION}

Elevated ALT and AST- the salient markers for non-alcoholic fatty liver disease with deranged dyslipidemia were found in long duration of known type 2 DM as well as in newly diagnosed diabetes subjects.

Copyright@ 23 May, 2021.

\section{REFERENCES}

1. IDF Diabetes Atlas $\mathbf{9}^{\text {th }}$ edition. 2019. Available at: https://www.diabetesatlas.org. [Last accessed on 2111-19]. 
2. Basit A, Fawwad A, Qureshi H, \& Shera AS. Prevalence of diabetes, pre-diabetes and associated risk factors: Second National Diabetes Survey of Pakistan (NDSP), 2016-2017. BMJ open. 2018; 8(8):e02096170.

3. Mohamed J, Nafizah AN, Zariyantey AH, Budin SB. Mechanisms of diabetes-induced liver damage: The role of oxidative stress and inflammation. Sultan Qaboos University Medical Journal. 2016; 16(2):132-41.

4. Kashinakunti SV, Rangappa M, \& Kallaganada GS. Correlation between liver enzymes and lipid profile in type II diabetes mellitus-A case control study. IOSR J Biotech \& Biochem (IOSR-JBB). 2017; 3(5):0105.

5. Pardhe BD, Kapali OS, Mathias J, Bhetwal A, Shakya J, Shrestha P. Elevated liver transaminases and their association with metabolic syndrome in type 2 diabetic patients attending tertiary care hospital of Nepal. Clin Lipidol. 2018; 13(1):4-12.

6. Mirmiran P, Gaeini Z, Bahadoran Z, Azizi F. Elevated serum levels of aminotransferases in relation to unhealthy foods intake: Tehran lipid and glucose study. BMC Endocrin Disorders. 2019; 19(1):1-8.

7. Viswanathan V, Kadiri M, Medimpudi S, \& Kumpatla $\mathrm{S}$. Association of non-alcoholic fatty liver disease with diabetic microvascular and macrovascular complications in South Indian diabetic subjects? Int J Diabet Dev Ctries. 2010; 30(4):208-212.

8. Al-Jameil N, Khan FA, Arjumand S, Khan MF, \& Tabassum $H$. Associated liver enzymes with hyperlipidemic profile in type 2 diabetes patients. Int $\mathrm{J}$ Clin Exp Pathol. 2014; 7(7):4345-9.

9. Alzahrani SH, Baig M, Bashawri JI, Aashi MM, Shaibi FK, \& Alqarni DA. Prevalence and Association of Elevated Liver Transaminases in Type 2 Diabetes Mellitus Patients in Jeddah, Saudi Arabia. Cureus. 2019; 11(7):1-8.

10. Wang YL, Koh WP, Yuan JM, \& Pan A. Association between liver enzymes and incident type 2 diabetes in Singapore Chinese men and women. BMJ Open Diabetes Res Care. 2016; 4(1):e000296.

11. World Health Organization. Definition and diagnosis of diabetes mellitus and intermediate hyperglycemia: report of a WHO/IDF consultation. WHO.2006.
12. Lee M, Kim M, Park JS, Lee S, You J, Ahn CW, et al. Higher glucagon-to-insulin ratio is associated with elevated glycated hemoglobin levels in type 2 diabetes patients. Korean J Intern Med. 2019; 34(5):1068-77.

13. Mandal A, Bhattarai $B$, Kafle $P$, Khalid $M$, Jonnadula SK, Lamicchane J, et al. Elevated Liver Enzymes in Patients with Type 2 Diabetes Mellitus and Nonalcoholic Fatty Liver Disease. Cureus. 2018; 10(11):19.

14. Cho $\mathrm{NH}$, Jang $\mathrm{HC}$, Choi $\mathrm{SH}$, Kim HR, Lee HK, Chan $\mathrm{JC}$, et al. Abnormal liver function test predicts type 2 diabetes: A community-based prospective study. Diabetes care. 2007; 30(10):2566-8.

15. Chandrashekhar GS. Alterations of liver enzymes in T2DM: A case control study. International Journal of Advances in Medicine. 2018; 5(6), 1521-1527.

16. Abro MUR, Butt A, Baqa K, Waris N, Khalid M, \& Fawwad A. Association of serum liver enzyme Alanine Aminotransferase (ALT) in patients with type 2 diabetes. Pak J Med Sci. 2018; 34(4):839-842.

17. Adhikari IP, Choudhury I, Anand P, Bhatia S, Tiwari R. Assessment of enzymatic markers in patients of chronic alcoholic liver disease attending OPD of a tertiary health care level Institute of North India. Int J Medi Health Res. 2019; 5(1):31-35.

18. Ni H, Soe HHK, \& Htet A. Determinants of abnormal liver function tests in diabetes patients in Myanmar. Int J Diab Res. 2012; 1(3):36-41.

19. Saligram S, Williams EJ, \& Masding MG. Raised liver enzymes in newly diagnosed Type 2 diabetes are associated with weight and lipids, but not glycemic control. Ind J Endocrin Metab. 2012; 16(6):1012-1014.

20. Bora K, Borah M, Chutia H, Nath CK, Das D, Ruram AA. Presence of concurrent derangements of liver function tests in type 2 diabetes and their relationship with glycemic status: A retrospective observational study from Meghalaya. J lab physicians. 2016; 8(1):3037.

21. Kalra S, Vithalani M, Gulati G, Kulkarni CM, Kadam Y, Pallivathukkal J, et al. Study of prevalence of nonalcoholic fatty liver disease (NAFLD) in type 2 diabetes patients in India (SPRINT). J Assoc Physicians India. 2013; 61(7):448-53. 


\section{AUTHORSHIP AND CONTRIBUTION DECLARATION}

\begin{tabular}{|c|l|l|l|}
\hline No. & Author(s) Full Name & \multicolumn{1}{|c|}{ Contribution to the paper } & Author(s) Signature \\
\hline 1 & Nazish Waris & $\begin{array}{l}\text { Concept and design, interpretation } \\
\text { of data and wrote the manuscript. } \\
\text { Concept and design, edited and } \\
\text { approved the final manuscript. } \\
\text { Concept and design, edited, } \\
\text { reviewed and approval of the final } \\
\text { manuscript. } \\
\text { Reviewed and approval the final } \\
\text { manuscript. }\end{array}$ \\
\hline 4 & Abdul Basit & Asher Fawwad & \\
\hline
\end{tabular}

